\title{
Pollos de engorde alimentados con harina de cayeno (Hibiscus rosa- sinensis)
}

\section{Broilers fed with cayeno (Hibiscus rosa-sinensis) flour}

\author{
Roa Vega María Ligia \\ Zootecnista. Esp. MSc. Docente de la Universidad de los Llanos \\ mroa@unillanos.edu.co
}

Recibido 10 de Mayo 2016, Aceptado 29 de Octubre 2016

\section{RESUMEN}

Este trabajo se llevó a cabo en Villavicencio Meta (Colombia), con el propósito de estudiar la inclusión de diferentes niveles de harina de Hibiscus rosa-sinensis (HCA) $(0,5,8$ y $12 \%)$ en dietas completas para pollos de engorde, reemplazando en parte otros alimentos de uso tradicional, principalmente la harina de carne y torta de soya. El diseño estadístico que se utilizó fue uno completamente al azar con cuatro tratamientos, tres replicaciones y 10 unidades experimentales en cada replicación ( $4 \times 3 \times 10=120$ aves). Se evaluó el aumento de peso, consumo, conversión alimenticia, valor biológico de la proteína (VB), coeficiente de eficiencia proteica (CEP), digestibilidad de la fibra en detergente neutro (FDN) y rendimiento en canal. El mayor aumento de peso diario por ave $(P<0.01)$ se observó en el tratamiento con $12 \%$ de cayeno, el cual fue $63.8 \mathrm{~g}$, mientras que el consumo diario fue más elevado $(\mathrm{P}<0.01)$ en la dieta con $8 \% 152 \mathrm{~g}$, siendo el más bajo en el del nivel $5 \%$ de HCA $119.6 \mathrm{~g}$. Las conversiones fueron mejores $(P<0.01)$ con el 5 y $12 \%$ de $H$. rosa-sinensis (2.22 y 2.29 respectivamente). Se presentó un incremento de la digestibilidad de la fibra detergente neutro (FDN) a medida que se elevaron los niveles de HCA en las dietas. El valor biológico más bajo $(P<0.01)$ se observó en la dieta en la que no se incluyó HCA (65.35\%), mientras que el rendimiento en canal fue mayor $(\mathrm{P}<0.01)$ para el tratamiento con $8 \%$ de $\mathrm{HCA}$ en comparación con los otros tres tratamientos. Se concluye que HCA se puede reemplazar en las dietas para pollos de engorde hasta en un $12 \%$, puesto que no afectó negativamente las variables analizadas en este trabajo, y sí se observó un 
mejoramiento de las mismas proporcional a los niveles de incremento de esta arbustiva. Es de resaltar que las canales de las aves alimentadas con HCA, presentaron una coloración amarilla y mejor gustosidad en comparación con el testigo.

Palabras clave: Avicultura, arbustivas, fibra, suplemento.

\begin{abstract}
This work was carried out in Villavicencio (Meta), with the purpose of studying the inclusion of different levels of flour of Hibiscus rosa-sinensis (CAF) (0, 5, 8 and $12 \%$ ) in diets for chickens fattening, partly replacing traditional use other foods, mainly meat meal and soybean meal. The statistical design used was a completely randomized with four treatments, three replications and 10 experimental units in each replication ( $4 \times 3 \times 10=120$ birds). Was evaluated weight gain, consumption, alimentary conversion, protein biological value (BV), protein efficiency ratio (PER), digestibility of neutral detergent fiber (NDF) and carcass yield. The biggest daily gain by bird $(P<0.01)$ was observed in the treatment with $12 \% \mathrm{CAF}$, which was $63.8 \mathrm{~g}$, while daily consumption was higher $(P<0.01)$ in the diet with $8 \% 152 \mathrm{~g}$, being the lowest level of $5 \%$ of CAF $119.6 \mathrm{~g}$. The conversions were better $(\mathrm{P}<0.01)$ with 5 and $12 \%$ of $H$. rosa-sinensis (2.22 and 2.29 respectively). Was presented an increase of digestibility of neutral detergent fiber (NDF) as HCA levels were elevated in the diets. The lowest biological value $(P<0.01)$ was observed in the diet in which no CAF $(65.35 \%)$, while the carcass yield was greater $(P<0.01)$ for treatment with $8 \% \mathrm{CAF}$ in comparison to the other three treatments. It is concluded that CAF can be replaced in diets for broilers up to $12 \%$, since it did not adversely affect the variables analyzed in this work, and was observed an improvement of them proportional to the levels of increase in this shrub. Is noteworthy that carcasses of birds fed CAF, presented a yellow coloration and better palatability compared with the control.
\end{abstract}

Keywords: Poultry, shrubby, fiber, supplement. 


\section{RESUMO}

Este trabalho foi realizado em Villavicencio (Meta), com a finalidade de estudar a inclusão de diferentes níveis de farinha de Hibiscus rosa-sinensis (HCA) (0. 5, 87 $12 \%$ ) em dietas completas para frangos de corte, substituindo em parte outros alimentos tradicionalmente utilizados, principalmente farinha de carne e farelo de soja. O delineamento estatístico utilizado foi o completamente aleatório com quatro tratamentos, três repetições e 10 unidades experimentais em cada replicação ( $4 \times 3 \times 10=120$ aves). Foi avaliada ganho de peso, consumo, conversão alimentar, proteína valor biológico (BV), taxa de eficiência protéica (CEP), a digestibilidade da fibra em detergente neutro (FDN) e rendimento de carcaça. O maior ganho de peso diário por ave $(\mathrm{P}<0.01)$ foi observada no tratamento com $12 \%$ de $H$. rosa-sinensis o que foi $63.8 \mathrm{~g}$, enquanto que 0 consumo diário foi maior $(\mathrm{P}<0.01)$ na dieta com $8 \% 152 \mathrm{~g}$, sendo o menor nível $5 \%$ de HCA $119.6 \mathrm{~g}$. As conversões foram melhores $(\mathrm{P}<0.01)$ com 5 e $12 \%$ de $H$. rosa-sinenss (2.22 e 2.29, respectivamente). Se apresentou um aumento da digestibilidade da fibra em detergente neutro (NDF) à medida que a ascensão los niveles de HCA nas dietas. O menor valor biológico $(\mathrm{P}<0.01)$ se observou na dieta em que não foi incluído HCA (65.35\%), enquanto que o rendimento de carcaça foi maior $(\mathrm{P}<0.01)$ para o tratamento com $8 \%$ HCA em comparação com as outras três tratamentos. Se conclui que o HCA pode substituir em as dietas para frangos de corte até um $12 \%$, desde que não alterem adversament as variáveis analisadas neste trabalho, e sim se observou um aperfeiçoamento da mesma proporcional ao aumento dos níveis deste arbusto. É digno de nota que as carcaças de aves alimentadas com HCA, apresentaram uma coloração amarela e melhor palatabilidade em comparação com o controlo.

Palavras-chave: Avicultura, arbustivas, fibra, suplemento.

\section{INTRODUCCIÓN}

Indudablemente la producción avícola ha tenido gran influencia técnica y económica en el sector agropecuario de Colombia en los últimos años, mostrando 
una gran capacidad de expansión, por el aumento de la demanda de carne y huevos, siendo en gran medida una de las principales fuentes de proteína para la población Colombiana ( $\mathrm{CCl}$ y MADR, 2011). Adicionalmente, se proyecta como industria internacional si se tiene el debido acompañamiento de las autoridades sanitarias, que con el esfuerzo de las empresas avícolas, se puede lograr la apertura de nuevos mercados en el exterior. Como resultado de este desempeño, la avicultura ocupa el segundo lugar dentro de las actividades agropecuarias en el país después de la ganadería de carne y de leche, siendo su participación aproximada del $11 \%$ del PIB. Se estima que más de 250.000 personas en 300 municipios, derivan su sustento de la cadena avícola conformada por la producción de material genético, carne y huevos, requiriendo de la agricultura para la alimentación de las aves como: maíz, soya, sorgo, principalmente (Martínez, 2012).

Para una excelente producción con pollos de engorde es necesario suplir adecuadamente los requerimientos de proteína y energía (Cuca et al., 2009), esto se logra utilizando ingredientes que aporten estos nutrientes teniendo cuidado de suministrar aminoácidos esenciales con el fin de permitir la formación de tejido muscular. Las dietas balanceadas incrementan ostensiblemente los costos de producción, por tanto buscar alternativas en las que se utilicen otros alimentos más económicos y que además proporcionen todos los nutrientes, es un reto para el sector avícola.

En la zona del piedemonte llanero existe una gran variedad de especies de árboles y arbustivas que tienen potencial para ser incorporadas en los sistemas de producción de animal, y en el caso de aves el principal factor limitante es la presencia de factores de fibra (Roa y Galeano, 2015). No obstante, la inclusión del follaje de arbustivas en la alimentación de aves mejora la presentación de la canal por el contenido de carotenos (Ku-Vera et al., 2000; Sosa et al., 2004). El cayeno (Hibiscus rosa-sinensis), es una arbustiva de crecimiento rápido, utilizada principalmente como planta de ornato. Según análisis nutricionales realizados por Roa et al., (2010) el cayeno contiene en porcentaje: 24.7 de materia seca (MS), 
13.8 proteína $(\mathrm{PC}), 1.8$ grasa, 11.1 fibra cruda $(\mathrm{FC}), 58.1$ extracto no nitrogenado (ENN), 9.5 cenizas, 20.4 fibra detergente neutro (FDN) y 17.4 fibra detergente ácido (FDA), con una digestibilidad in vitro de la materia seca (DIVMS) superior al $70 \%$. Su uso en la alimentación de aves ha sido poco estudiado, por lo tanto, esta investigación se basó en evaluar el comportamiento de pollos de engorde utilizando la arbustiva cayeno (Hibiscus rosa-sinensis), para lo cual se valoró la calidad de la proteína de las dietas de acuerdo a los métodos biológicos reportados por Church y Pond, (2002) con el fin de observar la respuesta del animal en función al consumo de proteína y la capacidad de retención de nitrógeno para sus actividades metabólicas. Para realizar estas evaluaciones se determinó el consumo y excreción de nitrógeno.

\section{METODOLOGIA}

Este trabajo se llevó a cabo, en la granja de la universidad de los Llanos y en laboratorio de nutrición animal, ubicados en Villavicencio (Meta); esta zona tiene una altitud $465 \mathrm{msnm}$, una temperatura promedio de $27^{\circ} \mathrm{C}$ y una precipitación anual entre 1900 a 2300 mm (IDEAM, 2013).

El experimento se realizó con 120 pollos de engorde de la línea genética Ross, de un día de nacidos siendo alimentados hasta los 20 días de edad con concentrado comercial de iniciación, luego se les ofreció dietas balanceadas las cuales fueron isoprotéicas (21\%) e isoenergéticas (2900 kcal de energía metabolizable)/kg de materia seca, a las que se incluyeron diferentes niveles de harina de cayeno (Hibiscus rosa-sinensis) (HCA) de la siguiente manera: Testigo T0 $=0 \%$ de $\mathrm{HCA}$, $\mathrm{T} 1=5 \%$ de HCA, $\mathrm{T} 2=8 \%$ de HCA, T3 $=12 \%$ de HCA. En la Tabla 1 se presentan los ingredientes utilizados en los tratamientos hasta los 45 días; durante 5 días se hizo un acostumbramiento de las aves a estas dietas.

Las aves se alojaron en jaulas con piso en malla, con el fin de facilitar la recolección de las excretas para valorar el porcentaje de digestibilidad de la fibra detergente neutro (FDN), y la excreción de proteína empleando el método de laboratorio Microkjeldalh. Con esta información se determinó el porcentaje de valor 
biológico (VB) de la proteína de las dietas experimentales. Con los datos de peso se calculó la conversión alimenticia y el coeficiente de eficiencia proteica (CEP), este último con el fin de establecer la cantidad de la proteína que utilizan las aves en la formación de los tejidos musculares. Las formulas aplicadas fueron tomadas de Church y Pond, (2002):

$$
\begin{gathered}
\% \mathrm{VB}=\frac{\text { Nitrogeno consumido }- \text { Nitrogeno excretado }}{\text { Nitrogeno consumido }} \times 100 \\
C E P=\frac{\text { Ganancia de peso }}{\text { Proteína consumioda }} \\
\% \text { de digestibilidad de FDN }=\frac{\text { FDN consumida }- \text { FDN excretada }}{\text { FDN consumida }} \times 100
\end{gathered}
$$

\begin{tabular}{|c|c|c|c|c|}
\hline \multirow{2}{*}{ INGREDIENTES (\%) } & \multicolumn{4}{|c|}{ TRATAMIENTOS } \\
\hline & TO & T1 & T2 & T3 \\
\hline Harina de Carne & 12.0 & 10.0 & 8.0 & 5.0 \\
\hline Torta de Soya & 17.0 & 17.0 & 17.0 & 17.0 \\
\hline Hibiscus rosa-sinensis & 0.0 & 5.0 & 8.0 & 12.0 \\
\hline Harina de Arroz & 20.0 & 19.3 & 18.1 & 17.0 \\
\hline Sorgo & 20.0 & 20.0 & 20.0 & 20.0 \\
\hline Maíz & 21.0 & 20.0 & 20.0 & 20.0 \\
\hline Melaza & 5.0 & 3.0 & 3.0 & 3.0 \\
\hline Aceite de Palma & 2.8 & 3.4 & 3.4 & 3.4 \\
\hline Otros Nutrientes & 2.2 & 2.3 & 2.5 & 2.6 \\
\hline Total & 100.0 & 100.0 & 100.0 & 100.0 \\
\hline \multicolumn{5}{|c|}{$\begin{array}{l}\text { Harina de cayeno (Hibiscus rosa-sinensis) }=(\mathrm{HCA}) \text {. Testigo }(\mathrm{T} 0)=0 \% \text { de HCA, } \mathrm{T} 1=5 \% \text { de HCA, } \\
\mathrm{T} 2=8 \% \text { de HCA y } \mathrm{T} 3=12 \% \text { de HCA. }\end{array}$} \\
\hline
\end{tabular}

Tabla 1. Ingredientes utilizados en dietas con cayeno (Hibiscus rosa-sinensis) en pollos de engorde entre 20-45 días de edad. 
( $4 \times 3 \times 10=120$ aves) y se evaluó: aumento de peso, consumo de alimento, conversión alimenticia, valor biológico (VB) de la proteína, coeficiente de eficiencia proteica (CEP), digestibilidad de la fibra detergente neutro (FDN) y rendimiento en canal, para establecer diferencias se aplicó prueba de comparación de medias Duncan. El modelo estadístico fue el siguiente:

$$
Y i j=\mu+\tau i+\varepsilon i j
$$

En donde, $Y i j$ es la variable respuesta de la $i j$-esima unidad experimental. $\mu$ es el efecto de la media general. $\tau i$ es el efecto del $i$-esimo tratamiento (T0, T1, T2 y T3). $\varepsilon i j$ es el efecto del error experimental asociado a la $i$-esima unidad experimental.

\section{RESULTADOS Y DISCUSIÓN}

Los resultados de los análisis nutricionales fueron similares para los cuatro tratamientos en lo relacionado a proteína y extracto no nitrogenado, mientras que la grasa fue menor en T2 y T3 en comparación con T0 y T1 (Tabla 2); lo contrario sucedió con el contenido de fibra cruda y FDN, observándose un mayor porcentaje en T2 y T4. Con relación a la composición nutricional de la harina de $\mathrm{H}$. rosasinensis, su contenido en porcentaje es: Proteína (14.2 \pm 0.6$)$, grasa $(2.3 \pm 0.1)$, fibra cruda $(13.1 \pm 0.9)$ y FDN $(23.7 \pm 3.9)$ fueron levemente superiores a lo reportado por Roa et al., (2010) 13.8, 1.8, 11.1 y 20.4\% respectivamente. Se ha demostrado que el medio ambiente, época del año, calidad de suelo y fertilización tienen una gran influencia en la calidad nutricional de los forrajes, por tanto, estas diferencias en los valores de proteína, grasa, fibra cruda y FDN, se pueden dar en un mismo forraje en este caso el cayeno, porque siempre va a depender de los factores mencionados (Sosa et al., 2004).

Aunque las dietas fueron isoproteicas, el consumo de este nutriente fue superior $(\mathrm{P}<0.01)$ en T3 y T4, donde la inclusión de cayeno fue mayor, igual sucedió con la excreción de materia seca (MS), nitrógeno total y FDN (Tabla 3), lo cual se explica por el mayor contenido de FDN de estos dos tratamientos, debido al aporte adicional del cayeno, lo que promueve la digestión bacteriana de la fibra en los 
ciegos y por tanto el desarrollo de estos órganos, como se evidenció cuando se sacrificaron los pollos. Esto concuerda con los resultados de lji et al., (2001), quienes analizaron el tracto digestivo de las aves, definiendo que los ciegos se originan en la unión del intestino delgado y el recto y se extienden oralmente hacia el hígado, y que el suministro de dietas con buen contenido de celulosa puede modificar el valor nutritivo de las mismas mediante diversos mecanismos, entre ellos merecen destacarse sus efectos sobre el desarrollo anatómico del tracto intestinal, sobre la velocidad de tránsito digestivo, su capacidad de intercambio iónico y su potencial como sustrato para la fermentación microbiana.

Tabla 2. Composición nutricional de las dietas con cayeno (Hibiscus rosa-sinensis) en pollos de engorde entre 20-45 días de edad.

\begin{tabular}{|c|c|c|c|c|c|}
\hline \multirow{2}{*}{$\begin{array}{l}\text { NUTRIENTES } \\
(\%)\end{array}$} & \multirow{2}{*}{ HCA } & \multicolumn{4}{|c|}{ TRATAMIENTOS } \\
\hline & & TO & T1 & T2 & T3 \\
\hline Proteína & $14.2 \pm 0.6$ & $21.0 \pm 0.4$ & $21.0 \pm 0.5$ & $21.0 \pm 0.3$ & $21.0 \pm 0.4$ \\
\hline Grasa & $2.3 \pm 0.1$ & $5.29 \pm 0.2$ & $5.22 \pm 0.1$ & $3.13 \pm 0.2$ & $3.24 \pm 0.2$ \\
\hline Fibra Cruda & $13.1 \pm 0.9$ & $4.67 \pm 0.3$ & $4.25 \pm 0.2$ & $6.12 \pm 0.5$ & $6.32 \pm 0.4$ \\
\hline ENN & $57.8 \pm 3.7$ & $60.7 \pm 4.9$ & $61.3 \pm 4.5$ & $60.2 \pm 4.3$ & $62.1 \pm 4.7$ \\
\hline Cenizas & $9.8 \pm 1.7$ & $8.8 \pm 1.3$ & $9.1 \pm 1.4$ & $9.5 \pm 1.0$ & $10.0 \pm 1.9$ \\
\hline FDN & $23.7 \pm 3.9$ & $25.0 \pm 4.1$ & $24.0 \pm 3.5$ & $28.9 \pm 3.8$ & $26.8 \pm 4.4$ \\
\hline \multicolumn{6}{|c|}{$\begin{array}{l}\text { El aumento de peso total y diario por ave fue mayor ( } \mathrm{P}<0.01) \text { en T3 en } \\
\text { comparación con los demás tratamientos, siendo el de menor aumento el testigo } \\
\text { (Tabla 4) en los periodos en los cuales se realizaron los pesajes, de } 20 \text { a } 45 \text { días } \\
\text { de edad T1, T2 y T3 mostraron un comportamiento similar; a los } 45 \text { días T3 } \\
\text { demostró un mayor aumento de peso, siendo el nivel más alto de HCA (Gráfica 1). } \\
\text { Resultados diferentes reportó Roa, (2011), utilizando cuatro niveles ( } 0,5,8 \text { y 12\%) } \\
\text { de harinas de Trichanthera gigantea y Erythrina poeppigiana en experimentos } \\
\text { independientes utilizando pollos de engorde, en los cuales se observó una } \\
\text { disminución en la ganancia de peso cuando se incrementaba la cantidad de harina } \\
\text { de estos dos forrajes en la dieta con respecto al testigo. }\end{array}$} \\
\hline
\end{tabular}


Tabla 3. Datos experimentales obtenidos con pollos de engorde alimentados con varios niveles de cayeno (Hibiscus rosa-sinensis)

\begin{tabular}{lcccc}
\hline \multirow{2}{*}{\multicolumn{1}{c}{ PARAMETROS }} & \multicolumn{4}{c}{ TRATAMIENTOS } \\
\cline { 2 - 5 } & T0 & T1 & T2 & T3 \\
\hline Consumo de proteína x ave/día (g) & $27.06^{\mathrm{a}}$ & $25.11^{\mathrm{a}}$ & $32.53^{\mathrm{b}}$ & $30.71^{\mathrm{b}}$ \\
Consumo de nitrógeno x ave/día (g) & $4.33^{\mathrm{a}}$ & $4.02^{\mathrm{a}}$ & $5.20^{\mathrm{b}}$ & $4.91^{\mathrm{b}}$ \\
Nitrógeno en excretas (\%) & 4.50 & 3.72 & 3.68 & 4.05 \\
Excreción pollinaza x ave/día en base seca (g) & $33.45^{\mathrm{ab}}$ & $30.60^{\mathrm{a}}$ & $40.20^{\mathrm{b}}$ & $37.50^{\mathrm{b}}$ \\
Nitrógeno excretado x ave/día (g) & $1.5^{\mathrm{b}}$ & $1.14^{\mathrm{a}}$ & $1.48^{\mathrm{b}}$ & $1.52^{\mathrm{b}}$ \\
FDN consumida x ave/día (g) & 31.30 & 28.70 & 43.93 & 39.20 \\
FDN en excretas (\%) & 43.00 & 45.00 & 49.10 & 46.00 \\
FDN excretada x ave/día (g) & $14.38^{\mathrm{a}}$ & $13.77^{\mathrm{a}}$ & $19.73^{\mathrm{b}}$ & $17.25^{\mathrm{ab}}$ \\
\hline
\end{tabular}

Letras distintas indican diferencias entre tratamiento $(\mathrm{P}>0.01)$. $\mathrm{FDN}=$ Fibra detergente neutro. Harina de cayeno (Hibiscus rosa-sinensis) $=(\mathrm{HCA})$ Testigo $(\mathrm{TO})=0 \%$ de $\mathrm{HCA}, \mathrm{T} 1=5 \%$ de $\mathrm{HCA}$, $\mathrm{T} 2=8 \%$ de $\mathrm{HCA}$ y $\mathrm{T} 3=12 \%$ de HCA.

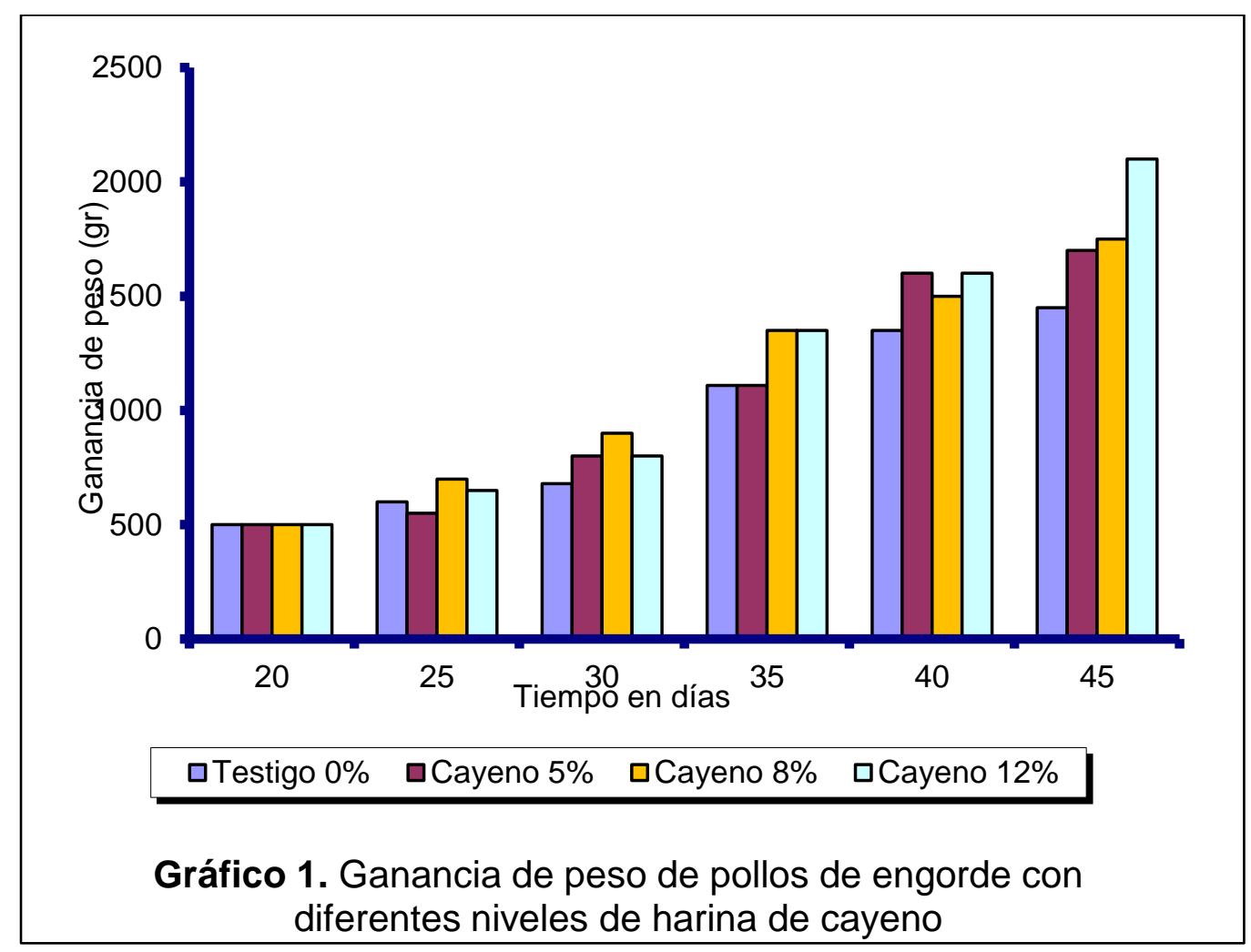

En cuanto a los consumos se incrementaron $(\mathrm{P}<0.01)$ cuando se suministraron los tratamientos con harina de cayeno al 8 y 12\% (Tabla 4), mostrando en T2 un 
mayor consumo durante los periodos evaluados (Figura 2), presentando T1 el menor consumo, y con T3 la mejor conversión alimenticia $(\mathrm{P}<0.01)$ con relación a los otros dos tratamientos (Tabla 4). Lo contrario encontró Roa, (2011) reportando que la conversón alimenticia se disminuyó a medida que se incrementaron los niveles de $T$. gigantea y E. poeppigiana en las dietas.

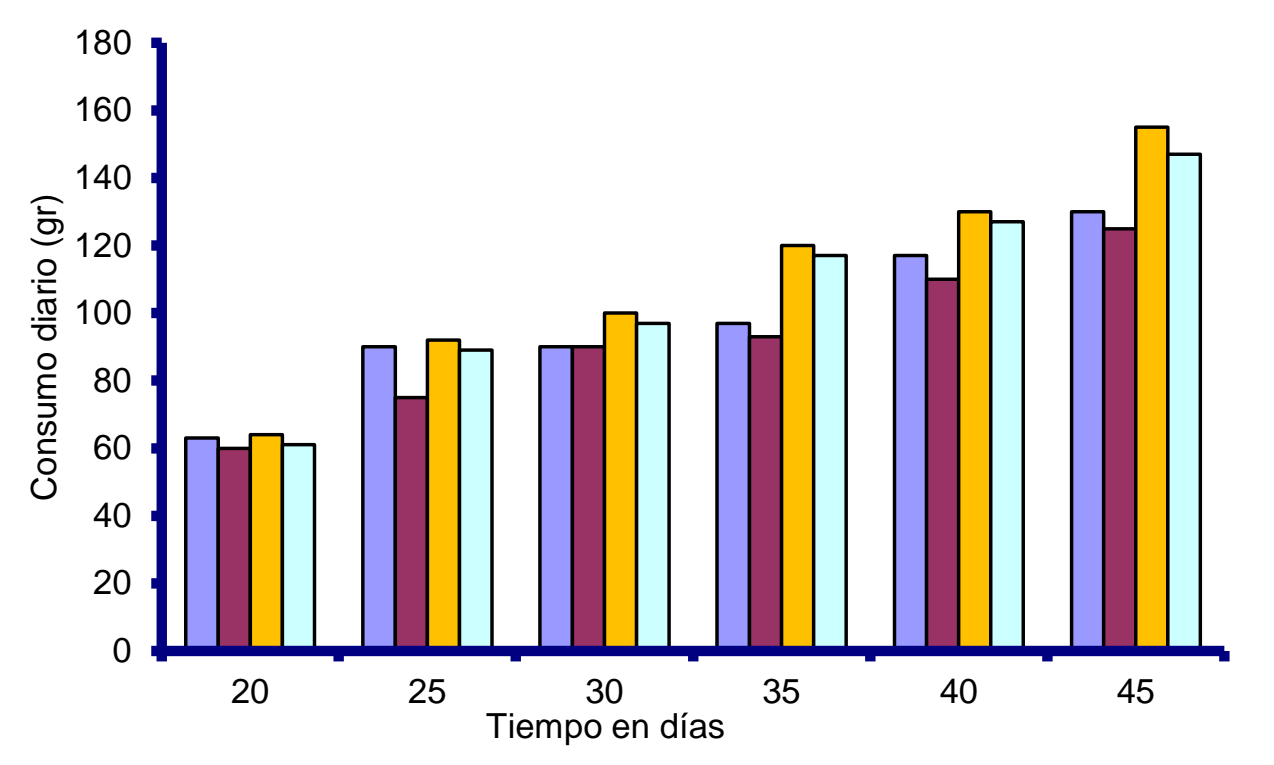

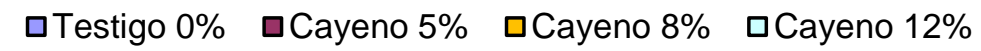

Gráfico 2. Consumo diario de pollos de engorde con diferentes niveles de harina de cayeno

Con respecto a la digestibilidad de la FDN se observó un incremento $(P<0.01)$ a medida que se elevaron los niveles de inclusión de cayeno (Tabla 4). Esto significa que la sustancia gelatinosa que contiene esta arbustiva, reportada por Roa et al., (1999) no afectó en forma negativa el consumo, la palatabilidad y aprovechamiento de las dietas hasta el $12 \%$, aunque no se descarta la presencia de otros factores antinutricionales que pueden afectar estas variables cuando las raciones tengan niveles superiores al estudiado en este trabajo. Estos resultados de una mayor digestibilidad en T2 y T3 donde es mayor el contenido de HCA, se deben indudablemente al efecto que tiene la celulosa en el tracto digestivo de los 
animales, promoviendo el desarrollo de bacterias que ayudan a degradar la pared celular, tal como lo reporta Pérez, (2005).

Tabla 4. Evaluación de pollos de engorde alimentados con varios niveles de cayeno (Hibiscus rosa-sinensis)

\begin{tabular}{lcccc}
\hline \multirow{2}{*}{\multicolumn{1}{c}{ VARIABLES }} & \multicolumn{4}{c}{ TRATAMIENTOS } \\
\cline { 2 - 5 } & T0 & T1 & T2 & T3 \\
\hline Días de experimentación & 25 & 25 & 25 & 25 \\
Peso promedio inicial/ave (g) & $500 \pm 23$ & $500 \pm 23$ & $500 \pm 23$ & $500 \pm 23$ \\
Peso final/ave (g) & 1559.8 & 1845.3 & 1873.8 & 2095.0 \\
Aumento de peso total/ave (g) & $1059.8^{\mathrm{c}}$ & $1345.3^{\mathrm{b}}$ & $1373.8^{\mathrm{b}}$ & $1595.0^{\mathrm{a}}$ \\
Aumento de peso ave/día (g) & $42.39^{\mathrm{c}}$ & $53.81^{\mathrm{b}}$ & $54.95^{\mathrm{b}}$ & $63.8^{\mathrm{a}}$ \\
Consumo ave/día (g) & $125.22^{\mathrm{c}}$ & $119.6^{\mathrm{d}}$ & $152.0^{\mathrm{a}}$ & $146.25^{\mathrm{b}}$ \\
Conversión (Consumo/peso) & $2.95^{\mathrm{a}}$ & $2.22^{\mathrm{b}}$ & $2.77 \mathrm{a}$ & $2.29^{\mathrm{b}}$ \\
Valor Biológico (\%) & $65.35^{\mathrm{c}}$ & $71.57^{\mathrm{a}}$ & $71.53^{\mathrm{a}}$ & $69.0^{\mathrm{b}}$ \\
Coeficiente de eficiencia proteica & $1.57^{\mathrm{b}}$ & $2.14^{\mathrm{a}}$ & $1.69^{\mathrm{b}}$ & $2.07^{\mathrm{a}}$ \\
(peso/consumo proteína) & $54.0^{\mathrm{c}}$ & $52.0^{\mathrm{d}}$ & $55.1^{\mathrm{b}}$ & $56.0^{\mathrm{a}}$ \\
Digestibilidad de FDN (\%) & $68.26^{\mathrm{a}}$ & $66.75^{\mathrm{ab}}$ & $65.16^{\mathrm{b}}$ & $66.15^{\mathrm{ab}}$ \\
\hline Rendimiento en canal (\%) & & & &
\end{tabular}

Letras distintas indican diferencias entre tratamiento $(\mathrm{P}>0.01)$ harina de cayeno (Hibiscus rosasinensis $)=(\mathrm{HCA})$. Testigo $(\mathrm{T0})=0 \%$ de $\mathrm{HCA}$ y $\mathrm{T} 1=5 \%$ de $\mathrm{HCA}, \mathrm{T} 2=8 \%$ de $\mathrm{HCA}, \mathrm{T} 3=12 \%$ de HCA.

En el valor biológico (VB) y el coeficiente de eficiencia proteica (CEP) (Tabla 4), se observaron diferencias $(P<0.01)$, siendo superior en estas dos variables $\mathrm{T} 1$ (71.57\% y 2.14 respectivamente), a parir de lo cual se puede deducir que haciendo reemplazos del $5 \%$ con HCA en las dietas para pollos de engorde no se afecta la calidad de la proteína en la dieta porque tiene un buen aprovechamiento biológico para las funciones metabólicas.

Los rendimientos en canal se disminuyeron con la inclusión de 8 y 12\% de HCA (Tabla 4), siendo mejor la del testigo $(\mathrm{P}<0.01)$, aunque la carne de los pollos alimentados con dietas que contenían HCA presentaron un color amarillo y un sabor culinario agradable cuando se prepararon y se ofrecieron a la degustación, característica que se puede tener en cuenta para su mercadeo. 


\section{CONCLUSIONES}

La harina de cayeno (Hibiscus rosa-sinensis) se puede incluir hasta en un $12 \%$ en dietas para pollos de engorde con el fin de reemplazar fuentes de proteína como harina de carne y torta de soya, sin que se afecte negativamente el consumo de alimento, aumento de peso, conversión, valor biológico de la proteína y digestibilidad de la fibra detergente neutro. Al contrario, se observó un mejoramiento de estas variables cuando se incrementaron los niveles de HCA en la dieta.

Es importante señalar que las canales de las aves alimentadas con cayeno, presentaron una coloración amarilla y mejor gustosidad en comparación con el testigo.

\section{REFERENCIAS BIBLIOGRÁFICAS}

1. AOAC. Official methods of analysis of AOAC International. Association of Official Analytical Chemists. Washington DC, USA, Ed: $18^{\text {th }} .746$ p. 2005.

2. Bermúdez R., Roa ML. Uso de harina de hojas de morera (Morus alba) en la alimentación de codornices en postura (Coturnix coturnix japónica. Revista Sistemas de Producción Agroecológicos, 4 (2): 2-13. 2013.

3. Corporación Colombia Internacional (CCl), Ministerio de Agricultura y Desarrollo Rural (MADR). Resultados encuesta planta de sacrifico de pollo, 2011A, Reportes de la oferta agropecuario, Sistema de información de la oferta agropecuaria. 2011.

4. Cuca G, Ávila E, Pró A. Alimentación de las aves. Departamento De Zootecnia, Dirección de patronato Universitario, Universidad Autónoma Chapingo. México. 276 p. 2009.

5. Church DC, Pond WG. Fundamentos de nutrición y alimentación de animales. Limusa, Wiley. México. D.F. 635 p. 2002.

6. Instituto de Hidrología, Meteorología y Estudios Ambientales (IDEAM). Información Histórica, Climatografía de las principales ciudades, Cartas Climatológicas - Medidas Mensuales, Aeropuerto Vanguardia. 2014. Recuperado $15 \quad$ Enero 2015. Disponible En: http://bart.ideam.gov.co/cliciu/villavo/tabla.htm

7. Iji PA, Saki A, Tivey DR. Body and intestinal growth of broiler chicks on a commercial starter diet.1. Intestinal weigh and mucosal development. British Poultry Science, 42 (4): 505-513. 2001.

8. Ku-Vera JC, Ramírez-Avilés L, Jiménez-Ferrer G, Alayón JA, RamírezCancino L. Árboles y arbustos para la producción animal en el trópico mexicano. En: Sánchez MD y Rosales M (Eds). Agroforestería para la Producción Animal en Latinoamérica. FAO, Roma. p 161-180. 2000. 
9. Martínez E. Avicultores prevén una década de crecimiento. En: Portafolio, Edición del 2 de Octubre del 2012. p 9.

10. National Research Council (NRC). Nutrient Requirements of Poultry. Ninth Revised Edition. National Academy Press. Washington DC. 155 p. 1994.

11. Pérez C. Alimentación de gallinas en pastoreo con morera (Morus alba), nacedero (Trichanthera gigantea) y concentrado comercial en la hacienda Vegas de la Clara, Gómez Plata, Antioquia. Tesis Médico Veterinario, Universidad de Antioquia. 2005.

12. Roa ML, Céspedes DA, Muñoz J. Evaluación Nutricional de tres especies de Árboles Forrajeros en Bovinos. Revista ACOVEZ, 21 (2): 14-18. 1999.

13. Roa ML, Castillo CA, Téllez E. Influencia del tiempo de maduración en la calidad nutricional de ensilajes con forrajes arbóreos. Revista Sistemas de Producción Agroecológicos.1 (1): 63-73. 2010.

14. Roa ML. Pollos alimentados con diferentes niveles de harina de Trichanthera gigantea y Erythrina poeppigiana. Revista Sistemas de producción Agroecológicos, 2 (1): 22-33. 2011.

15. Roa ML, Galeano J. Calidad nutricional y digestibilidad in situ de ensilajes de cuatro leñosas forrajeras. Pastos y Forrajes, 38 (4): 431-440. 2015.

16. Rostagno S, Teixeira L, Lopes J, Gomes P, De Oliveira F, Lopes D, Ferreira A, De Toledo $S$ y Euclides R. Tablas brasileras para pollos y cerdos. Universidad de Federal de Viçosa-Departamento de Zootecnia, 251 p. 2011.

17. Sosa E, Pérez D, Ortega L, Zapata G. Evaluación del potencial forrajero de árboles y arbustos tropicales para la alimentación de ovinos. Técnica Pecuaria en México, 42 (2): 129-14. 2004. 